\title{
Ondas planas em um meio estratificado
}

\author{
Nascimento, J. O. do ${ }^{1^{*}}$; Pereira, H. B. B ${ }^{1,2}$; Moreira, D. M ${ }^{1}$; Moret, M. $A^{1,2}$ \\ 1 Programa de Modelagem Computacional, CIMATEC, Salvador, BA, Brasil. \\ 2 Universidade do Estado da Bahia, Salvador, Bahia, Brasil.
}

* e-mail: jeffersonascimento@gmail.com

\begin{abstract}
Resumo
Conforme literatura científica, bem como as obras específicas de Rijo, um meio estratificado é um meio constituído por várias camadas horizontais. Para analisar como os campos elétrico e magnético se comportam num meio estratificado, é necessário estudar tal comportamento em um meio formado por uma única camada demasiadamente espessa de tal forma que podemos considerá-la um meio ilimitado. O presente artigo tem como objetivo apresentar de forma direta e didática, visando um público que deseja se inserir e iniciar seus estudos em Geofísica, na linha de pesquisa de Métodos Eletromagnéticos (utilizando ou não modelagem computacional), apresentando um formalismo físico-matemático da supracitada temática. Demonstrar que a propagação do campo elétrico e magnético (nas condições citadas) podem ser descritos por meio de uma equação de onda. Partindo das considerações de um meio ilimitado, homogêneo e isotrópico e, apresentado os percursos metodológicos; conforme os alicerces fornecidos pelas Equações de Maxwell, o presente estudo apresenta como resultado a equação de onda denominada de Equação de Helmholtz.
\end{abstract}

Palavras-chave: Equação de Helmholtz, Equação da Onda, Métodos Eletromagnéticos, Geofísica.

\begin{abstract}
According to scientific literature, as well as the specific works of Rijo, a stratified medium is a medium consisting of several horizontal layers. In order to analyze how the electric and magnetic fields behave in a stratified medium, it is necessary to study such behavior in a medium formed by a single layer too thick in such a way that we can consider it an unlimited medium. The aim of this article is to present a direct and didactic approach, aiming at an audience that wishes to insert and begin their studies in Geophysics, in the line of research of Electromagnetic Methods (using or not computational modeling), presenting a physicalmathematical formalism of the aforementioned thematic Demonstrate that the propagation of the electric and magnetic field (in the mentioned conditions) can be described by means of a wave equation. Starting from the considerations of an unlimited, homogeneous and isotropic medium and, presented the methodological paths; according to the foundations provided by Maxwell's Equations, the present study presents as result the wave equation called the Helmholtz Equation
\end{abstract}

Keywords: Helmholtz equation, Wave Equation, Electromagnetic Methods, Geophysics.

\section{Introdução}

Na obra elaborada por Rijo [1] é apresentada a definição para um meio estratificado. É aquele constituído por várias camadas horizontais. Em caráter inicial de pesquisa, um meio estratificado pode ser considerado como sendo o que dispõe de uma camada extremamente espessa, e assim, podemos fazer a consideração dela ser ilimitada [1].

Nesta camada ilimitada admitiremos também que o meio seja homogêneo e isotrópico. Estas três considerações são fundamentais para a condução da proposta apresentada aqui. Por meio delas, consideramos que os campos elétrico e magnético apresentam variação apenas na direção z.

Este artigo tem como objetivo apresentar de forma didática, clara, direta e objetiva que as equações dos campos elétrico e magnético correspondem a equações de ondas, sendo planas e uniformes no espaço, representadas pela Equação de Helmholtz [1].

Acreditamos que pela exposição didática aqui apresentada, o presente artigo poderá ajudar aos discentes a nível de pós-graduação em geofísica, na área de métodos eletromagnéticos a entenderem estes resultados iniciais. Ressaltamos por último que este 
artigo corresponde a uma homenagem, em especial do primeiro autor a um antigo professor seu que não se encontra mais entre nós: o professor Luiz Rijo.

\section{Método e Resultados}

A metodologia apresentada na presenta pesquisa inicia a partir das Equações de Maxwell. A Lei de Gauss (Eq. 1), Lei de Gauss para o magnetismo ou a conservação de fluxo magnético (Eq. 2), Lei de Faraday (Eq. 3) e a Lei de Ampère (Eq. 4):

$$
\begin{aligned}
& \nabla \cdot D=\rho \\
& \nabla \cdot B=0 \\
& \nabla \times E=-\frac{\partial B}{\partial t} \\
& \nabla \times H=J+\frac{\partial D}{\partial t}
\end{aligned}
$$

Utilizando a Eq. 4 e substituindo os valores correspondentes para a densidade de corrente $\mathrm{J}$ e da indução elétrica $D$, teremos:

$\nabla \times H=\sigma E+\varepsilon \frac{\partial E}{\partial t}$. Transformando a equação anterior, passando do domínio do tempo para a frequência, por meio da transformada de Fourier, encontraremos:

$\nabla \times H=\sigma E+i \omega \varepsilon E$. Deixando o campo elétrico em evidência obteremos:

$\nabla \times H=(\sigma+i \omega \varepsilon) E$. A soma dos termos que multiplicam o campo elétrico pode ser substituída pela admitividade $\eta$, em que obteremos a Eq. 5:

$$
\nabla \times H=\eta E
$$

Resolvendo o rotacional na Eq. 5, da seguinte forma:

$$
\nabla \times H=\left(\frac{\partial}{\partial x} i+\frac{\partial}{\partial y} j+\frac{\partial}{\partial z} k\right) \times\left(H_{x}+H_{y}+H_{z}\right)
$$

e

$$
\nabla \times H=\left|\begin{array}{ccc}
i & j & k \\
\frac{\partial}{\partial x} & \frac{\partial}{\partial y} & \frac{\partial}{\partial z} \\
H_{x} & H_{y} & H_{z}
\end{array}\right|
$$

Encontraremos o seguinte resultado para o rotacional:

$$
\nabla \times H=\left(\frac{\partial H_{z}}{\partial y}-\frac{\partial H_{y}}{\partial z}\right) i+\left(\frac{\partial H_{x}}{\partial z}-\frac{\partial H_{z}}{\partial x}\right) j+\left(\frac{\partial H_{y}}{\partial x}-\frac{\partial H_{x}}{\partial y}\right) k
$$

Ao substituirmos o resultado anterior na Eq. 5, ela passará ao seguinte formato:

$$
\left(\frac{\partial H_{z}}{\partial y}-\frac{\partial H_{y}}{\partial z}\right) i+\left(\frac{\partial H_{x}}{\partial z}-\frac{\partial H_{z}}{\partial x}\right) j+\left(\frac{\partial H_{y}}{\partial x}-\frac{\partial H_{x}}{\partial y}\right) k=\eta E
$$

Lembrando que em um meio ilimitado, homogêneo e isotrópico a variação do campo ocorre na direção z. Assim, utilizando o termo do eixo das abscissas que satisfazem a condição anterior, teremos:

$-\frac{\partial H_{y}}{\partial z}=\eta E_{y}$, obtermos a Eq. a seguir:

$$
\frac{\partial H_{y}}{\partial z}+\eta E_{x}=0
$$

Satisfazendo ainda a condição para a variação do campo na direção $z$, utilizando o termo na direção y da Eq. 6, obteremos:

$\frac{\partial H_{x}}{\partial z}=\eta E_{y}$, passando ao formato da seguinte equação:

$$
\frac{\partial H_{x}}{\partial z}-\eta E_{y}=0
$$

Utilizaremos a Eq. 8 posteriormente. Retornando ao pensamento da Eq. 7 e isolando o campo elétrico na direção $\mathrm{x},-\eta E_{x}=\frac{\partial H_{y}}{\partial z}$, encontraremos a seguinte equação:

$$
E_{x}=-\frac{1}{\eta} \frac{\partial H_{y}}{\partial z}
$$

Passaremos agora a utilizar a Eq. 3, a Lei de Faraday e, substituindo o equivalente na indução magnética, teremos: 
$\nabla \times E=-\mu \frac{\partial H}{\partial t}$. Repetindo o pensamento inicial utilizado e passando a equação anterior para o domínio da frequência, teremos:

$$
\nabla \times E=-i \omega \mu H \text { ou } \nabla \times E=-Z H
$$

$\mathrm{Na}$ Eq. 10 o novo termo que substitui os anteriores após a mudança de domínio, é chamado de impeditividade $(\boldsymbol{Z})$. Resolveremos o rotacional na Eq. 10 da seguinte maneira:

$$
\nabla \times E=\left(\frac{\partial}{\partial x} i+\frac{\partial}{\partial y} j+\frac{\partial}{\partial z} k\right) \times\left(E_{x}+E_{y}+E_{z}\right),
$$

logo:

$\nabla \times E=\left(\frac{\partial E_{z}}{\partial y}-\frac{\partial E_{y}}{\partial z}\right) i+\left(\frac{\partial E_{x}}{\partial z}-\frac{\partial E_{z}}{\partial x}\right) j+\left(\frac{\partial E_{y}}{\partial x}-\frac{\partial E_{x}}{\partial y}\right) k$

Por meio do resultado anterior a Eq. 10 terá o seguinte formato:

$$
\left(\frac{\partial E_{z}}{\partial y}-\frac{\partial E_{y}}{\partial z}\right) i+\left(\frac{\partial E_{x}}{\partial z}-\frac{\partial E_{z}}{\partial x}\right) j+\left(\frac{\partial E_{y}}{\partial x}-\frac{\partial E_{x}}{\partial y}\right) k=-Z H
$$

Como vimos a variação do campo elétrico (semelhante ao magnético) será na direção z. Desta forma, teremos $-\frac{\partial E_{y}}{\partial z}=-Z H_{x}$, que pode ser reescrita da seguinte maneira:

$$
\frac{\partial E_{y}}{\partial z}-Z H_{x}=0
$$

Na direção y o termo que varia em z, teremos:

$$
\frac{\partial E_{x}}{\partial z}=-Z H_{z} \text { ou } \frac{\partial E_{x}}{\partial z}+Z H_{y}=0
$$

Isolando o campo magnético na Eq. 12, chegaremos ao seguinte resultado:

$$
Z H_{x}=\frac{\partial E_{y}}{\partial z} \rightarrow H_{x}=\frac{1}{Z} \frac{\partial E_{y}}{\partial z}
$$

Retomando a Eq. 13 e substituindo nela o campo elétrico da Eq. 9, teremos

$$
\frac{\partial}{\partial z}\left(-\frac{1}{\eta} \frac{\partial H_{y}}{\partial z}\right)+Z H_{y}=0 \rightarrow-\frac{1}{\eta} \frac{\partial^{2} H_{y}}{\partial z^{2}}+Z H_{y}=0
$$

Multiplicando os termos da equação anterior pela admitividade, a equação anterior passará a ter o seguinte formato:

$$
\frac{\partial^{2} H_{y}}{\partial z^{2}}-Z \eta H_{y}=0, \quad \text { utilizando } \quad k^{2}=-Z \eta, \quad \text { e }
$$
lembrando que só há variação dos campos na direção z, teremos:

$$
\frac{\partial^{2} H_{y}}{\partial z^{2}}+k^{2} H_{y}=0 \rightarrow \frac{d^{2} H_{y}}{d z^{2}}+k^{2} H_{y}=0
$$

Retomando a Eq. 8 e substituindo o valor do campo magnético da Eq. 14, encontraremos a seguinte equação:

$$
\frac{\partial}{\partial z}\left(\frac{1}{Z} \frac{\partial E_{y}}{\partial z}\right)-\eta E_{y}=0 \rightarrow \frac{1}{Z} \frac{\partial^{2} E_{y}}{\partial z^{2}}-\eta E_{y}=0 .
$$

Multiplicando a equação anterior pela impeditividade $(Z)$, resultará em: $\frac{\partial^{2} E_{y}}{\partial z^{2}}-Z \eta E_{y}=0$. Substituindo $k^{2}=-Z \eta$, chegaremos ao seguinte resultado:

$\frac{\partial^{2} E_{y}}{\partial z^{2}}+k^{2} E_{y}=0 . \quad$ Lembrando das condições consideradas, teremos:

$$
\frac{d^{2} E_{y}}{d z^{2}}+k^{2} E_{y}=0
$$


A Eq. 16 corresponde a Equação de Helmholtz, cuja solução é a apresentada abaixo.

$$
E_{y}(z)=E_{0} e^{-i k|z|}\left\{\begin{array}{l}
E_{0} e^{-i k z}, z>0 \\
E_{0} e^{i k z}, \quad z<0
\end{array}\right.
$$

A Eq. 15 também corresponde a Equação de Helmholtz, de solução análoga a anterior:

$$
H_{y}(z)=H_{0} e^{-i k|z|}\left\{\begin{array}{l}
H_{0} e^{-i k z}, z>0 \\
H_{0} e^{i k z}, z<0
\end{array}\right.
$$

\section{Considerações finais}

Ao observarmos as soluções da Equação de Helmholtz, há uma variação que ocorre de forma senoidal no tempo e no espaço [1]. Uma função que varia, simultaneamente, no tempo e no espaço é chamada de onda, independentemente se a variação for senoidal ou não. Desta forma a equação de Helmholtz corresponde a uma equação da onda. Conforme Rijo [1] para os resultados apresentados a onda é denominada de plana uniforme. Ela é plana pelo fato da frente de onda ser paralela ao plano xy [2]. Pelas condições consideras, para um meio ilimitado, homogêneo e isotrópico, a onda variar apenas na direção $z$, é que faz com que seja chamada de uniforme [1].

\section{Agradecimentos}

Jefferson Nascimento agradece à FAPESB por meio do apoio financeiro parcial recebido através da bolsa de doutorado (BOL170/2015). Marcelo A. Moret agradece ao CNPq pelo suporte financeiro parcial oriundo de sua bolsa de Produtividade em Pesquisa (No. 304454/2014-1).

Este artigo é uma Homenagem Póstuma ao professor Rijo.

\section{Referências}

[1] RIJO, L. ELECTRICAL GEOPHYSICS 1-D Earth Direct Modeling. UFPA, Belém, 2004. 